\title{
EVALUACIÓN COMPARTIDA EN LA CREACIÓN Y REPRESENTACIÓN DE UNA COMPOSICIÓN TEATRAL EN LA ASIGNATURA DE EDUCACIÓN FÍSICA EN EDUCACIÓN SECUNDARIA
}

Shared evaluation in the creation and representation of a theatrical composition in the subject of Physical Education

Avaliação partilhada na criação e representação de uma composição teatral na disciplina de Educação Física na educação secundária

\section{Daniel Bores García (1)}

(1) Universidad Rey Juan Carlos, España. Teléfono: +34 605788305. Correo electrónico: daniel.bores@urjc.es

\section{Resumen}

En el presente trabajo se expone una experiencia escolar en la que se utiliza la evaluación compartida mediante una escala de valoración en una unidad didáctica perteneciente al bloque expresivo con 253 alumnos de $1^{\circ} \mathrm{ESO}$ en la asignatura de Educación Física. Se realiza también un salto a la calificación, otorgando a cada uno de los agentes evaluadores (autoevaluación, coevaluación y heteroevaluación) un máximo de un punto que contribuirá a la nota de la segunda evaluación del curso académico. Los resultados obtenidos muestran unos buenos resultados, algo más bajos en los tres grupos no bilingües. En cuanto a la diferencia en la evaluación triádica, se observan mayores puntuaciones en la autoevaluación, seguidas por la heteroevaluación. Los procesos de coevaluación dan lugar a puntuaciones más bajas.

Palabras clave: Evaluación compartida; Educación Física; Escala de valoración

\begin{abstract}
In this work we present a didactic experience in which shared assessment was used by means of a rating scale in a didactic unit belonging to the expressive block, with 253 students of the first year of Compulsory Secondary Education (1 ${ }^{\circ}$ ESO) in Physical Education. We also focus on grades, giving for each of the assessing agents (selfassessment, peer assessment and hetero-assessment) a maximum of one point that will contribute to the grade of the second quarter of the academic year. The results obtained


show good results, somewhat lower in the three non-bilingual groups. Regarding the difference in the triadic assessment, higher scores were observed in the self-assessment, followed by the hetero-assessment. The peer assessment processes achieved lower scores.

Keywords: Shared assessment; Physical Education; Rating scale

\section{Resumo}

No presente trabalho é apresentada uma experiência escolar em que se utiliza a avaliação partilhada por meio de uma escala de classificação numa unidade didática pertencente ao bloco expressivo que tem 253 alunos do $1^{\circ} \mathrm{ESO}$ na disciplina de Educação Física. Realizou-se também uma análise à classificação, atribuindo a cada um dos agentes avaliadores (autoavaliação, coavaliação e heteroavaliação) um máximo de um ponto que contribuirá para a nota da segunda avaliação do ano letivo. Os resultados obtidos mostram bons resultados, sendo um pouco piores nos três grupos não bilíngues. Em relação à diferença na avaliação triádica, observam-se maiores pontuações na autoavaliação, seguida da heteroavaliação. Os processos de coavaliação originaram pontuações mais baixas.

Palavras-chave: Avaliação partilhada; Educação Física; Escala de classificação

\section{Introducción}

Se expone una experiencia escolar en la que se utiliza la evaluación compartida mediante el uso de una escala de valoración en una Unidad Didáctica (UD) de expresión corporal llevada a cabo con 253 alumnos de $1^{\circ} \mathrm{ESO}$. La escala de valoración, inspirada en la propuesta de Hernando, Hortigüela \& Pérez-Pueyo (2017), se utiliza durante el proceso de creación y ensayo de una representación final grupal basada en la expresión corporal y como instrumento de calificación de la Unidad Didáctica, usando la evaluación triádica (autoevaluación, coevaluación y heteroevaluación).

\section{Contextualización}

La experiencia narrada a continuación es parte de la UD "Uso mi cuerpo para contar historias" perteneciente a la programación de $1^{\circ} \mathrm{ESO}$ de la asignatura de Educación Física y temporalizada en la segunda evaluación del curso, entre los meses de enero y 
febrero. Esta UD se ha aplicado a nueve grupos del IES Alameda de Osuna, en el distrito de Barajas (Madrid). Un total de 253 alumnos han participado en la experiencia.

La UD tuvo una duración de cuatro semanas, con dos lecciones semanales de 55 minutos de duración. Durante las primeras tres sesiones el profesor propuso una serie de actividades de imitación, representación y expresión, en las que los alumnos trabajaron de forma individual en primer lugar y después en pequeños grupos. Durante las lecciones 4, 5 y 6 los alumnos crearon y ensayaron sus representaciones finales por grupos y en la lección 7 tuvo lugar la exposición de las mismas, momento en el cual tuvo lugar el proceso de calificación que después se expone. El criterio de evaluación relacionado con esta UD es, entonces, "interpretar y reproducir de acciones motrices con finalidades artístico-expresivas, utilizando técnicas de expresión corporal y otros recursos" (Decreto 48/2015, de 14 de mayo, p.221) concretado en el estándar de aprendizaje 2.1. del mismo Decreto, relativo a la utilización de técnicas corporales de manera creativa.

\section{Diseño y desarrollo}

La Tabla 1 muestra la progresión seguida en la UD durante las siete sesiones.

Tabla 1.

Diseño de la UD

\begin{tabular}{ll}
\hline FASES DE LA UD & \multicolumn{1}{c}{ EXPLICACIÓN DE LAS FASES } \\
\hline $\begin{array}{l}\text { 1.Actividades de } \\
\text { familiarización con la } \\
\text { expresión corporal }\end{array}$ & $\begin{array}{l}\text { Tres lecciones con actividades orientadas a trabajar aspectos básicos de la } \\
\text { expresión corporal tales como la imitación, la representación y la expresión. }\end{array}$ \\
$\begin{array}{l}\text { 2.Creación y ensayo de } \\
\text { la representación final }\end{array}$ & $\begin{array}{l}\text { Tras una breve explicación de la escala de valoración, los alumnos elijen sus } \\
\text { propias agrupaciones siguiendo los criterios de número de miembros (4-5) y } \\
\text { agrupamientos mixtos. Disponen de tres lecciones completas para la creación y } \\
\text { el ensayo de la representación final. }\end{array}$ \\
$\begin{array}{l}\text { 3.Representación final } \\
\text { y utilización de la } \\
\text { escala de valoración }\end{array}$ & $\begin{array}{l}\text { Los alumnos, por grupos, hacen sus representaciones finales. Al término de } \\
\text { cada una de ellas, tiene lugar la evaluación triádica, usando tres copias de la } \\
\text { misma escala de valoración (una para el grupo, otra para el profesor y otra para } \\
\text { el resto de grupos). }\end{array}$ \\
\hline
\end{tabular}

La Figura 1 muestra la escala de valoración utilizada como instrumento de evaluación, que amplía, detallándolo al mismo tiempo, el estándar de aprendizaje señalado en el epígrafe anterior. En la presente experiencia esta representación se califica con una contribución de hasta 3 puntos en la nota de la segunda evaluación. 
Hasta un máximo de un punto es logrado a partir de cada uno de los tres agentes de la evaluación triádica (el propio grupo, el resto de grupos y el profesor).

Figura 1.

Escala de valoración para la representación final

\begin{tabular}{|c|c|c|c|}
\hline \multicolumn{4}{|c|}{ Nombre y apellidos de los miembros del grupo: } \\
\hline \multicolumn{4}{|c|}{$\begin{array}{l}\text { Requisitos previos: grupos de } 4-5 \text { alumnos (mixtos). Todos los participantes se involucrarán en el proceso creativo, en los } \\
\text { ensayos y en la representación. Se dedicarán } 3 \text { clases a la creación y ensayo de la representación y una clase más para } \\
\text { la representación final. }\end{array}$} \\
\hline $\begin{array}{l}\text { Elementos } \\
\text { valorables }\end{array}$ & Subvalor & Criterios de logro & $\begin{array}{l}\text { Máximo } \\
\text { valor }\end{array}$ \\
\hline \multirow[t]{5}{*}{$\begin{array}{l}\text { Creación del } \\
\text { Storyboard }\end{array}$} & 15 & $\begin{array}{l}\text { El storyboard tiene buenos dibujos y recoge los elementos más importantes de } \\
\text { la representación. }\end{array}$ & \multirow[t]{5}{*}{15} \\
\hline & 10 & $\begin{array}{l}\text { El storyboard tiene buenos dibujos y recoge algunos elementos de la } \\
\text { representación. }\end{array}$ & \\
\hline & 10 & $\begin{array}{l}\text { El storyboard tiene dibujos poco trabajados y recoge los elementos más } \\
\text { importantes de la representación. }\end{array}$ & \\
\hline & 5 & $\begin{array}{l}\text { El storyboard tiene dibujos poco trabajados y recoge algunos elementos de la } \\
\text { representación. }\end{array}$ & \\
\hline & 0 & $\begin{array}{l}\text { El storyboard tiene dibujos muy deficientes y no aporta casi ninguna } \\
\text { información. }\end{array}$ & \\
\hline \multirow[t]{3}{*}{ Duración } & 10 & La representación dura entre 4 y 6 minutos & \multirow[t]{3}{*}{10} \\
\hline & 5 & La representación se desvía menos de 30 segundos del tiempo estipulado. & \\
\hline & 0 & La representación se desvía más de 20 segundos del tiempo estipulado. & \\
\hline \multirow{4}{*}{$\begin{array}{l}\text { Capacidad } \\
\text { narrativa }\end{array}$} & 20 & La historia se comprende perfectamente & \multirow[t]{4}{*}{20} \\
\hline & 12 & La historia se entiende, aunque no completamente & \\
\hline & 5 & $\begin{array}{l}\text { Hay muchos elementos que no se entienden, lo cual hace difícil seguir la } \\
\text { historia }\end{array}$ & \\
\hline & 0 & La historia es incomprensible & \\
\hline \multirow{4}{*}{$\begin{array}{l}\text { Capacidad } \\
\text { expresiva }\end{array}$} & 20 & La representación es muy rica en elementos corporales expresivos & \multirow[t]{4}{*}{20} \\
\hline & 12 & La representación tiene algunos elementos corporales expresivos & \\
\hline & 5 & La representación tiene pocos recursos expresivos interesantes & \\
\hline & 0 & La representación no tiene ningún recurso expresivo interesante & \\
\hline \multirow{4}{*}{$\begin{array}{l}\text { Capacidad } \\
\text { creativa }\end{array}$} & 20 & La representación es muy original y creativa & \multirow[t]{4}{*}{20} \\
\hline & 12 & La representación tiene algunos aspectos creativos interesantes & \\
\hline & 5 & La representación no tiene casi elementos creativos & \\
\hline & 0 & La representación no es ni original ni creativa & \\
\hline \multirow{3}{*}{$\begin{array}{l}\text { Fluidez } \\
\text { (coordinación) }\end{array}$} & 15 & Se observa coordinación y compenetración entre los miembros del grupo & \multirow[t]{3}{*}{15} \\
\hline & 8 & La coordinación y compenetración es mejorable & \\
\hline & 0 & $\begin{array}{l}\text { Los miembros del grupo actúan de manera descoordinada, sin } \\
\text { compenetración. }\end{array}$ & \\
\hline \multicolumn{3}{|c|}{ PUNTUACIÓN FINAL (máximo 100 puntos) } & \\
\hline \multicolumn{3}{|c|}{ APORTE PARA LA NOTA FINAL (puntos $\times$ 0,01) } & \\
\hline
\end{tabular}

Evaluación compartida en la creación y representación de una composición teatral en la asignatura de 


\section{Evaluación}

Una buena forma de evaluar la experiencia es a través de los datos obtenidos de la evaluación triádica de las representaciones finales de los nueve grupos. Como se ve en la Tabla 2, se obtienen en general unos buenos resultados (la mayor parte de los grupos superan los 150 puntos sobre 300), si bien son algo peores en los grupos no bilingües (A, B y C), hecho que concuerda con el rendimiento académico comparado con el resto de materias curriculares, en las que las calificaciones en exámenes y finalmente en las evaluaciones son significativamente más bajas que en los grupos bilingües. Como ya se ha señalado anteriormente, la puntuación final de cada grupo no será la media de las puntuaciones obtenidas a partir de los tres tipos de evaluación, sino la suma de las mismas hasta un máximo de 300 puntos (3 puntos sobre 10 en la nota de la asignatura en la $2^{\mathrm{a} E v a l u a c i o ́ n) . ~ H a c i e n d o ~ u n ~ a n a ́ l i s i s ~ p o r ~ t i p o ~ d e ~ e v a l u a c i o ́ n, ~ l a s ~ p u n t u a c i o n e s ~ m a ́ s ~}$ elevadas salen de los procesos de autoevaluación (72,6/100 puntos de promedio), dado el interés del alumnado, con poca experiencia previa en autoevaluación, en obtener una puntuación alta que pueda compensar las puntuaciones obtenidas mediante la coevaluación (López-Pastor, Barba-Martín \& González, 2005), seguidas por la heteroevaluación (62,2/100 puntos de promedio). En la coevaluación es donde observamos las puntuaciones más bajas en términos generales (57,3/100 puntos de promedio). En este tipo de evaluación el alumnado, con la inmadurez fruto de una falta de experiencia previa en la evaluación triádica como ya se ha señalado anteriormente (López-Pastor et al., 2005), entiende que de la coevaluación no va a obtener un beneficio para la nota de su propio grupo, por lo que es más exigente con el resto de grupos. Algunos de los alumnos, tras realizar la evaluación de los compañeros, reconocen haberse dejado influir en ciertas ocasiones por criterios ajenos a la propia representación, como pueden ser criterios de afinidad.

Tabla 2.

Puntuaciones a partir de la evaluación triádica

\begin{tabular}{|c|c|}
\hline TIPO DE EVALUACIÓN & PUNTUACIONES PROMEDIO POR GRUPO \\
\hline Autoevaluación & $\begin{array}{l}1^{\circ} \mathrm{A}=64,1^{\circ} \mathrm{B}=50,1^{\circ} \mathrm{C}=70,1^{\circ} \mathrm{D}=78,1^{\circ} \mathrm{E}=76,1^{\circ} \mathrm{F}=84,1^{\circ} \mathrm{G}=80,1^{\circ} \mathrm{H}=85,1^{\circ} \mathrm{I}=67 \\
\text { Puntuación promedio }=72,6\end{array}$ \\
\hline Coevaluación & $\begin{array}{l}1^{\circ} \mathrm{A}=39,1^{\circ} \mathrm{B}=29,1^{\circ} \mathrm{C}=58,1^{\circ} \mathrm{D}=56,1^{\circ} \mathrm{E}=65,1^{\circ} \mathrm{F}=72,1^{\circ} \mathrm{G}=58,1^{\circ} \mathrm{H}=76,1^{\circ} \mathrm{I}=63 \\
\text { Puntuación promedio }=57,3\end{array}$ \\
\hline Heteroevaluación & $\begin{array}{l}1^{\circ} \mathrm{A}=49,1^{\circ} \mathrm{B}=43,1^{\circ} \mathrm{C}=48,1^{\circ} \mathrm{D}=59,1^{\circ} \mathrm{E}=71,1^{\circ} \mathrm{F}=70,1^{\circ} \mathrm{G}=73,1^{\circ} \mathrm{H}=78,1^{\circ} \mathrm{I}=69 \\
\text { Puntuación promedio }=62,2\end{array}$ \\
\hline $\begin{array}{l}\text { Puntuación total } \\
\text { (sobre } 300)\end{array}$ & $\begin{array}{l}1^{\circ} \mathrm{A}=152, \quad 1^{\circ} \mathrm{B}=122, \quad 1^{\circ} \mathrm{C}=176, \quad 1^{\circ} \mathrm{D}=193, \quad 1^{\circ} \mathrm{E}=212, \quad 1^{\circ} \mathrm{F}=226, \quad 1^{\circ} \mathrm{G}=211, \\
1^{\circ} \mathrm{H}=239,1^{\circ} \mathrm{I}=19\end{array}$ \\
\hline
\end{tabular}

Evaluación compartida en la creación y representación de una composición teatral en la asignatura de 


\section{Transferibilidad de la experiencia}

Esta experiencia puede transferirse a otro tipo de contenidos en Educación Física, si bien deberá ser modificada en tanto en cuanto tanto el centro como el alumnado sean diferentes, ya que la escala de valoración posibilita la implicación del alumnado en el proceso evaluativo al hacerlo partícipe y consciente de su proceso de aprendizaje y actúa como instrumento eficaz en la evaluación compartida, permitiendo participar del proceso al alumnado, más allá de la tradicional figura del profesor como único agente evaluador (Hortigüela, Pérez-Pueyo \& Moreno, 2018).

\section{Referencias}

Decreto 47/2015, de 14 de mayo, del Consejo de Gobierno, por el que se establece para la Comunidad de Madrid el currículo de la Educación Secundaria Obligatoria. BOCM, 118, de 20 de mayo, 10-309. Recuperado de https://www.bocm.es/boletin/CM_Orden_BOCM/2015/...BOCM-20150520-

\section{$\underline{1 . P D F}$}

Hernando, A., Hortigüela, D., \& Pérez-Pueyo, A. (2017). El proceso de evaluación formativa en la realización de un video tutorial de estiramientos en inglés en un centro bilingüe. En López-Pastor y Pérez-Pueyo (Ed.), Evaluación formativa y compartida en educación: experiencias de éxito en todas las etapas educativas (pp. 260-270). León: Universidad de León.

Hortigüela, D., Pérez-Pueyo, A., \& Moreno, A. (2018). Evaluación formativa y compartida en la formación inicial del profesorado. Estudios Pedagógicos, 44(2), 7-8.

López-Pastor, V.M., Barba-Martín, J.J., \& González, M. (2005). La participación del alumnado en la evaluación: la autoevaluación, la coevaluación y la evaluación compartida. Tándem, 17, 21-37. 